\title{
Embeddings in Spacetimes Sourced by Scalar Fields
}

\author{
E. Anderson ${ }^{1}$, F. Dahia ${ }^{2}$, J. E. Lidsey ${ }^{3}$ and C. Romero ${ }^{4}$
}

${ }^{1}$ Astronomy Unit, School of Mathematical Sciences, Queen Mary, Mile End Road, London E1 4NS, U.K.

Departamento de Física, Universidade Federal da Paraíba, C. Postal 5008, João Pessoa, PB 58059-970, Brazil

\begin{abstract}
The extension of the Campbell-Magaard embedding theorem to general relativity with minimally-coupled scalar fields is formulated and proven. The result is applied to the case of a self-interacting scalar field for which new embeddings are found, and to Brans-Dicke theory. The relationship between Campbell-Magaard theorem and the general relativity Cauchy and initial value problems is outlined.
\end{abstract}




\section{Introduction}

There is currently a high level of interest in higher-dimensional theories of gravity, motivated in part by recent developments in both string theory and early-universe cosmology. There is a growing body of evidence [1] supporting the conjecture that the five perturbative, 10-d string theories may correspond to different limiting cases of a more fundamental, non-perturbative, 11-d 'M-theory', which reduces to 11-d supergravity in the infrared limit. (For recent reviews, see, e.g., Refs. 2]). Inspired by these advances, the proposal that our observable universe may be regarded as a domain wall or 'brane' that is embedded in a higher-dimensional space-time has recently become popular in early-universe cosmology [3, 4, 5, 6]. In the braneworld scenario, the back reaction of the brane results in a higher-dimensional geometry that is non-factorizable. Consequently this scenario is clearly distinct from the standard Kaluza-Klein compactification scheme. Indeed, in the five-dimensional Randall-Sundrum models, the extra dimension need not be compact [6].

An important question that arises in such braneworld scenarios is the relationship between the geometry of the apparent, lower-dimensional world and that of the embedding, higher-dimensional space-time. It is therefore important, in view of the above discussion, to develop embedding theorems that enable such questions to be concretely addressed (for an overview and extensive bibliography, see [7]). An important theorem is due to Campbell and Magaard (CM) [18, 19] and states that any $n$-dimensional, (semi-)Riemannian manifold $\left(M^{n}, g\right)$ can be locally and isometrically embedded in an $(n+1)$-dimensional manifold $\left(N^{n+1}, \tilde{g}\right)$, where the Ricci curvature of $N^{n+1}$ vanishes [18, 19]. The theorem was suggested by Campbell [18] and a proof was later offered by Magaard [19. The theorem has been discussed in a number of contexts in the literature [8, 9, 10, 11, 12, 13, 14, 15, 16, 17.

Recently, we showed [14, 15] how to extend this theorem to the class of embeddings where $\left(N^{n+1}, \tilde{g}\right)$ is an Einstein space, with a non-zero Ricci tensor that is directly proportional to the metric, $\tilde{g}$. Specific classes of embeddings, such as those of Einstein spaces within Einstein spaces, were established [14].

Einstein spaces can be viewed as solutions to General Relativity (GR), where the Ricci curvature is generated by a particular source - the cosmological constant. A natural question to address is whether further extensions of the CM theorem are possible and, in particular, to investigate embeddings in spaces that are sourced by dynamical matter fields. One of the simplest models for matter is a scalar field. In this paper, we extend the CM analysis to include a minimally-coupled scalar field with a general potential energy arising through its self-interactions, and for Brans-Dicke theory [20].

The paper is organized as follows. In Sec 2 we extend the CM theorem to spaces sourced by a scalar field, identifying the mathematical conditions that must be satisfied by such a field in order for the embedding of a given manifold to be possible in principle. In Sec 3.1, it is shown that minimally-coupled scalar self-interacting scalar fields satisfy these conditions. Examples of such embeddings are given in Sec 3.2. The Brans-Dicke field is then considered in Sec 3.3. We then outline the clarifying relation between the CM theorem and the GR Cauchy [21, 22, 23] and initial value [24, 25, 26] problems in Sec 4. We conclude in Sec 5.

\section{Campbell-Magaard Theorem with Scalar Field}

The proof of the Campbell-Magaard (CM) theorem 18, 19, 8, and of its extension to the case when the embedding manifold is an Einstein space [14, 15] follows a scheme similar to the methods employed when investigating the GR Cauchy Problem, i.e., once the initial conditions for the metric in a 3-dimensional hypersurface are given, one would like to know whether the Einstein field equations (EFE's) with a non-trivial source admit a unique solution.

In GR, the space-time metric is determined by the Einstein equations

$$
G_{\mu \nu}=-\kappa T_{\mu \nu},
$$

where $\kappa$ is the Einstein constant and $T_{\mu \nu}$ is the energy-momentum tensor, which is a function of the matter fields and the metric. We consider a scalar field $\bar{\chi}$ defined in a semi-Riemannian manifold ${ }^{1}\left(N^{n+1}, \tilde{g}_{\alpha \beta}\right)$. We assume that the energy-momentum tensor of this field is an analytic function of the field $\bar{\chi}$, its first derivatives and the metric tensor $\tilde{g}_{\alpha \beta}$ :

$$
\tilde{T}_{\mu \nu}=\tilde{T}_{\mu \nu}\left(\bar{\chi}, \frac{\partial \bar{\chi}}{\partial x^{\alpha}}, \tilde{g}_{\alpha \beta}\right)
$$

Let us choose a coordinate system in which the metric $\tilde{g}_{\alpha \beta}$ has the line element

$$
d s^{2}=\bar{g}_{i k} d x^{i} d x^{k}+\varepsilon \bar{\phi}^{2} d y^{2}
$$

\footnotetext{
${ }^{1}$ In this paper, Latin and Greek indices run from 1 to $n$ and 1 to $n+1$, respectively.
} 
where $y=x^{n+1}, \bar{\phi}=\bar{\phi}\left(x^{1}, \ldots, x^{n}, y\right)$ and $\bar{g}_{i k}=\bar{g}_{i k}\left(x^{1}, \ldots, x^{n}, y\right)$ and $\varepsilon= \pm 1$.

We suppose that the evolution of $\bar{\chi}$ is governed by a second-order p.d.e which may be written in the form

$$
\frac{\partial^{2} \bar{\chi}}{\partial y^{2}}=P\left(\bar{\chi}, \frac{\partial \bar{\chi}}{\partial x^{i}}, \frac{\partial \bar{\chi}}{\partial y}, \bar{g}_{\alpha \beta}, \frac{\partial \bar{g}_{\alpha \beta}}{\partial x^{i}}, \frac{\partial \bar{g}_{\alpha \beta}}{\partial y}, \frac{\partial^{2} \bar{\chi}}{\partial x^{i} \partial x^{k}}\right),
$$

where $P$ is analytic with respect to each of its arguments. We also make the physically reasonable assumption of energy-momentum conservation:

$$
\tilde{\nabla}_{\mu} \tilde{T}^{\mu \nu}=0 .
$$

$\tilde{\nabla}_{\mu}$ is the covariant derivative with respect to $\tilde{g}_{\mu \nu}$.

We now claim that if conditions (2), (4) and (5) hold, it is possible to locally embed any $n$-dimensional, (semi-) Riemannian manifold $\left(M^{n}, g\right)$ in a $(n+1)$-dimensional space sourced by the scalar field $\bar{\chi}$.

In the coordinates (3), the EFE's take the form

$$
\begin{aligned}
\tilde{R}_{i k} & =\bar{R}_{i k}+\varepsilon \bar{g}^{j m}\left(\bar{\Omega}_{i k} \bar{\Omega}_{j m}-2 \bar{\Omega}_{j k} \bar{\Omega}_{i m}\right)-\frac{\varepsilon}{\bar{\phi}} \frac{\partial \bar{\Omega}_{i k}}{\partial y}+\frac{1}{\bar{\phi}} \bar{\nabla}_{i} \bar{\nabla}_{k} \bar{\phi}=-\kappa\left(\tilde{T}_{i k}-\frac{1}{n-1} g_{i k} \tilde{T}\right), \\
\tilde{R}_{i}^{y} & =\frac{\varepsilon}{\bar{\phi}} \bar{g}^{j k}\left(\bar{\nabla}_{j} \bar{\Omega}_{i k}-\bar{\nabla}_{i} \bar{\Omega}_{j k}\right)=-\kappa \tilde{T}_{i}^{y}, \\
\tilde{G}_{y}^{y} & =-\frac{1}{2} \bar{g}^{i k} \bar{g}^{j m}\left(\bar{R}_{i j k m}+\varepsilon\left(\bar{\Omega}_{i k} \bar{\Omega}_{j m}-\bar{\Omega}_{j k} \bar{\Omega}_{i m}\right)\right)=-\kappa \tilde{T}_{y}^{y},
\end{aligned}
$$

where

$$
\bar{\Omega}_{i k}=-\frac{1}{2 \bar{\phi}} \frac{\partial \bar{g}_{i k}}{\partial y}
$$

and the barred terms are calculated with the metric $\bar{g}_{i k}$ induced on the hypersurface $\Sigma_{c}$ of constant $y=c$.

We now state the following lemma:

Lemma 1. Let the functions $\bar{g}_{i k}\left(x^{1}, \ldots, x^{n}, y\right), \bar{\phi}\left(x^{1}, \ldots, x^{n}, y\right)$ and $\bar{\chi}\left(x^{1}, \ldots, x^{n}, y\right)$ be analytic at $(0, \ldots, 0) \in$ $\Sigma_{0} \subset \Re^{n+1}$. Assume that the following conditions hold

i) $\bar{g}_{i k}=\bar{g}_{k i}$

ii) $\operatorname{det}\left(\bar{g}_{i k}\right) \neq 0$;

iii) $\bar{\phi} \neq 0$.

Assume further that $\bar{g}_{i k}$ and $\bar{\chi}$ satisfy the equations (4) and (6) in the open set $U \subset \Re^{n+1}$ which contains $0 \in \Re^{n+1}$ and (6) and (7) at $\Sigma_{0}$. Then, $\bar{g}_{i k}, \bar{\phi}$ and $\bar{\chi}$ satisfy (6) and (7) in a neighborhood of $0 \in \Re^{n+1}$.

Proof. The key point of the proof is given by (15). First, let us define the tensor $\tilde{F}_{\alpha \beta}=\tilde{G}_{\alpha \beta}+\kappa \tilde{T}_{\alpha \beta}$. By assumption, $\bar{\chi}$ satisfies (4) in a neighborhood $V \subset \Re^{n+1}$ of $0 \in \Re^{n+1}$, whence (5) also holds in $V$. It then follows that $\tilde{F}_{\alpha \beta}$ has vanishing divergence, so

$$
\frac{\partial \tilde{F}_{\beta}^{y}}{\partial y}=-\frac{\partial \tilde{F}_{\beta}^{i}}{\partial x^{i}}-\tilde{\Gamma}_{\mu \lambda}^{\mu} \tilde{F}_{\beta}^{\lambda}+\tilde{\Gamma}_{\lambda \beta}^{\mu} \tilde{F}_{\mu}^{\lambda} .
$$

On the other hand, by expressing the Einstein tensor in terms of the Ricci tensor, we can write

$$
\tilde{F}_{k}^{i}=\tilde{R}_{k}^{i}-\delta_{k}^{i}\left(\tilde{R}_{j}^{j}+\tilde{G}_{y}^{y}\right)+\kappa \tilde{T}_{k}^{i}
$$

Again, by assumption, the equation

$$
\tilde{R}_{k}^{i}=-\kappa\left[\tilde{T}_{k}^{i}-\frac{\delta_{j}^{i}}{n-1}\left(\tilde{T}_{j}^{j}+\tilde{T}_{y}^{y}\right)\right]
$$

holds in $V \subset \Re^{n+1}$ and it then follows that $\tilde{F}_{k}^{i}=-\delta_{k}^{i} \tilde{F}_{y}^{y}$ in $V$. After some algebra we may then deduce that

$$
\begin{aligned}
\frac{\partial \tilde{F}_{y}^{y}}{\partial y} & =-\varepsilon \bar{\phi}^{2} \bar{g}^{i j} \frac{\partial \tilde{F}_{i}^{y}}{\partial x^{j}}-2 \tilde{\Gamma}_{i y}^{i} \tilde{F}_{y}^{y}+\left(-\varepsilon \frac{\partial\left(\bar{\phi}^{2} \bar{g}^{i j}\right)}{\partial y^{j}}-\varepsilon \bar{\phi}^{2} \bar{g}^{i j} \tilde{\Gamma}_{k j}^{k}+\tilde{\Gamma}_{y y}^{i}\right) \tilde{F}_{i}^{y} \\
\frac{\partial \tilde{F}_{i}^{y}}{\partial y} & =\frac{\partial \tilde{F}_{y}^{y}}{\partial x^{i}}+2 \tilde{\Gamma}_{y i}^{y} \tilde{F}_{y}^{y}+\left(\tilde{\Gamma}_{y i}^{k}+\varepsilon \bar{\phi}^{2} \bar{g}^{k j} \tilde{\Gamma}_{i j}^{y}-\tilde{\Gamma}_{y \mu}^{\mu} \delta_{i}^{k}\right) \tilde{F}_{k}^{y}
\end{aligned}
$$

and since ([6) and (77) hold at the hypersurface $\Sigma_{0}$, it follows that $\tilde{F}_{\beta}^{y}=0$ and hence that $\partial \tilde{F}_{\beta}^{y} /\left.\partial y\right|_{\nu=0}=0$. 
It is not difficult to show by mathematical induction that all the derivatives of $\tilde{F}_{\beta}^{y}$ (to any order) vanish at $y=0$. As $\tilde{F}_{\beta}^{y}$ is analytic, we may therefore conclude that $\tilde{F}_{\beta}^{y}=0$ in an open set of $\Re^{n+1}$. Thus, (6) and (17) also hold in an open set of $\Re^{n+1}$ which includes the origin and this proves the lemma.

To proceed, we must now establish that the solutions $\bar{g}_{i k}, \bar{\phi}$ and $\bar{\chi}$ do indeed exist. With this in mind, let us now recall the Cauchy-Kowalewski (CK) theorem [27]:

Theorem (Cauchy-Kowalewski). Consider the set of partial differential equations:

$$
\frac{\partial^{2} u^{A}}{\partial\left(y^{n+1}\right)^{2}}=F^{A}\left(y^{\alpha}, u^{B}, \frac{\partial u^{B}}{\partial y^{\alpha}}, \frac{\partial^{2} u^{B}}{\partial y^{\alpha} \partial y^{i}},\right), \quad A=1, \ldots, m
$$

where $u^{1}, . ., u^{m}$ are $m$ unknown functions of the $n+1$ variables $y^{1}, \ldots, y^{n}, y^{n+1}, \alpha=1, \ldots, n+1, i=1, . ., n$, $B=1, \ldots, m$. Also, let $\xi^{1}, \ldots, \xi^{m}, \eta^{1}, \ldots, \eta^{m}$, be functions of the variables $y^{1}, \ldots, y^{n}$, and be analytic at $0 \in \Re^{n}$. If the functions $F^{A}$ are analytic with respect to each of their arguments around the values evaluated at the point $y^{1}=\ldots=y^{n}=0$, there exists a unique solution of equations (9) which is analytic at $0 \in \Re^{n+1}$ and that satisfies the initial conditions

$$
\begin{aligned}
u^{A}\left(y^{1}, \ldots, y^{n}, 0\right) & =\xi^{A}\left(y^{1}, \ldots, y^{n}\right) \\
\frac{\partial u^{A}}{\partial y^{n+1}}\left(y^{1}, \ldots, y^{n}, 0\right) & =\eta^{A}\left(y^{1}, \ldots, y^{n}\right), \quad A=1, \ldots, m .
\end{aligned}
$$

After solving ([6) for the second-order derivative of $\bar{g}_{i k}$ with respect to $y$ we find that

$$
\begin{aligned}
\frac{\partial^{2} \bar{g}_{i k}}{\partial y^{2}}= & -2 \varepsilon \kappa \bar{\phi}^{2}\left(T_{i k}-\frac{1}{n-1} g_{i k} T\right)+\frac{1}{\bar{\phi}} \frac{\partial \bar{\phi}}{\partial y} \frac{\partial \bar{g}_{i k}}{\partial y}-\frac{1}{2} \bar{g}^{j m}\left(\frac{\partial \bar{g}_{i k}}{\partial y} \frac{\partial \bar{g}_{j m}}{\partial y}-2 \frac{\partial \bar{g}_{i m}}{\partial y} \frac{\partial \bar{g}_{j k}}{\partial y}\right) \\
& -2 \varepsilon \bar{\phi}\left(\frac{\partial^{2} \bar{\phi}}{\partial x^{i} \partial x^{k}}-\frac{\partial \bar{\phi}}{\partial x^{j}} \bar{\Gamma}_{i k}^{j}\right)-2 \varepsilon \bar{\phi}^{2} \bar{R}_{i k} .
\end{aligned}
$$

Due to the symmetry of the tensors, $\bar{g}_{i k}$ and $T_{i k}$, we can rewrite (12) in terms of the components of $g_{i k}$ with $i \leq k$. This equation, together with the field equation (4), form a set of $1+n(n+1) / 2$ p.d.e's for the $1+n(n+1) / 2$ unknown functions, $\bar{g}_{i k}(i \leq k)$ and $\bar{\chi}$. (Note that $\bar{\phi}$ is a nonzero analytic function that is treated as a known).

Thus, the p.d.e system we have just obtained has the canonical form of (9) and, moreover, it satisfies all of the conditions required for the use of the CK theorem. Indeed, by virtue of the properties (2) and (4) imposed on $T_{\alpha \beta}$ and $P$, the right-hand side of the equations is comprised of functions of the variables

$$
x^{1}, . ., x^{n}, y ; \bar{g}_{i k}, \bar{\chi}, \frac{\partial \bar{g}_{i k}}{\partial x^{j}}, \frac{\partial \bar{g}_{i k}}{\partial y}, \frac{\partial \bar{\chi}}{\partial x^{j}}, \frac{\partial \bar{\chi}}{\partial y} ; \frac{\partial^{2} \bar{g}_{i k}}{\partial x^{j} \partial x^{m}}, \frac{\partial^{2} \bar{\chi}}{\partial x^{j} \partial x^{m}},
$$

which are analytic with respect to each of their arguments at

$$
x^{1}=0, . ., x^{n}=0, y=0 ;\left.\bar{g}_{i k}\right|_{0},\left.\bar{\chi}\right|_{0},\left.\frac{\partial \bar{g}_{i k}}{\partial x^{j}}\right|_{0},\left.\frac{\partial \bar{g}_{i k}}{\partial y}\right|_{0},\left.\frac{\partial \bar{\chi}}{\partial x^{j}}\right|_{0},\left.\frac{\partial \bar{\chi}}{\partial y}\right|_{0} ;\left.\frac{\partial^{2} \bar{g}_{i k}}{\partial x^{j} \partial x^{m}}\right|_{0},\left.\frac{\partial^{2} \bar{\chi}}{\partial x^{j} \partial x^{m}}\right|_{0},
$$

if $\left|\bar{g}_{i k}\right|_{0} \neq 0$. Therefore, given analytic initial conditions

$$
\begin{gathered}
\bar{g}_{i k}\left(x^{1}, . ., x^{n}, 0\right)=g_{i k}\left(x^{1}, . ., x^{n}\right), \quad \frac{\partial \bar{g}_{i k}}{\partial y}\left(x^{1}, . ., x^{n}, 0\right)=\bar{\phi}\left(x^{1}, . ., x^{n}, 0\right) \Omega_{i k}\left(x^{1}, \ldots, x^{n}\right) \\
\bar{\chi}\left(x^{1}, . ., x^{n}, 0\right)=\xi\left(x^{1}, . ., x^{n}\right), \frac{\partial \bar{\chi}}{\partial y}\left(x^{1}, . ., x^{n}, 0\right)=\eta\left(x^{1}, . ., x^{n}\right),
\end{gathered}
$$

which satisfy $\left|g_{i k}\right| \neq 0$, there exists a unique set of functions $\bar{g}_{i k}$ and $\bar{\chi}$ which solve the equations (4) and (6) which are analytic at the origin. It should be noted that an important feature of these solutions is the property $\left|\bar{g}_{i k}\right| \neq 0$ in some neighborhood of $0 \in \Re^{n+1}$.

If we take the initial conditions $g_{i k}$ as being the metric components of a (semi-) Riemannian space $M^{n}$ written in some coordinate system, then we can state the following theorem:

Theorem 1 Let $M^{n}$ be a n-dimensional, semi-Riemaninan manifold with metric given by $d s^{2}=g_{i k} d x^{i} d x^{k}$, in a coordinate system $\left\{x^{i}\right\}$ of $M^{n}$. Let $p \in M^{n}$ have coordinates $x_{p}^{1}=\ldots=x_{p}^{n}=0$. Then $M^{n}$ has a local isometric and analytic embedding (at the point $p$ ) in an $(n+1)$-dimensional space sourced by any arbitrary scalar field $\bar{\chi}$ that is characterized by the properties (2), (4) and (5) iff there exist functions $\Omega_{i k}\left(x^{1}, \ldots, x^{n}\right)(i, k=1, . ., n)$, $\xi\left(x^{1}, . ., x^{n}\right), \eta\left(x^{1}, . ., x^{n}\right)$ and $\phi\left(x^{1}, . ., x^{n}\right) \neq 0$ that are analytic at $0 \in \Re^{n}$ such that

$$
\begin{aligned}
\Omega_{i k} & =\Omega_{k i} \\
g^{j k}\left(\nabla_{j} \Omega_{i k}-\nabla_{i} \Omega_{j k}\right) & =-\varepsilon \kappa \phi T_{i}^{y}\left(\xi, \eta, g_{i j}\right) \\
q^{i k} q^{j m}\left(R_{i j k m}+\varepsilon\left(\Omega_{i k} \Omega_{j m}-\Omega_{j k} \Omega_{i m}\right)\right) & =2 \kappa T_{\nu}^{y}\left(\xi, \eta, q_{i j}\right) .
\end{aligned}
$$


Proof. $(\Rightarrow)$ If $M^{n}$ has an embedding in some arbitrary space sourced by a scalar field, then it can be proved [15, 28, that there exists a coordinate system in which the metric of the embedding space has the form $d s^{2}=\bar{g}_{i k} d x^{i} d x^{k}+\varepsilon \bar{\phi}^{2} d y^{2}$, where the analytic functions $\bar{g}_{i k}\left(x^{1}, \ldots, x^{n}, y\right)$ and $\bar{\phi}\left(x^{1}, \ldots, x^{n}, y\right)$ are such that $\bar{\phi}\left(x^{1}, \ldots, x^{n}, y\right) \neq 0$ and $\bar{g}_{i k}\left(x^{1}, \ldots, x^{n}, 0\right)=g_{i k}\left(x^{1}, \ldots, x^{n}\right)$ in an open set of $\Re^{n}$ which contains the origin. Given that the embedding space is, by assumption, generated by a scalar field $\bar{\chi}$, it follows that $\bar{g}_{i k}$ and $\bar{\phi}$ necessarily satisfy the equations (44), (6), (6) and (7) for some field $\bar{\chi}\left(x^{1}, \ldots, x^{n}, y\right)$ in a neighborhood of $0 \in \Re^{n+1}$. In particular, the equations (6) and (7) hold for $y=0$. Therefore, the functions $\Omega_{i k}\left(x^{1}, \ldots, x^{n}\right)(i, k=1, . ., n)$, $\xi\left(x^{1}, . ., x^{n}\right), \eta\left(x^{1}, . ., x^{n}\right)$ and $\phi\left(x^{1}, . ., x^{n}\right)$, as defined by

$$
\Omega_{i k}=\bar{\Omega}_{i k}\left(x^{1}, . ., x^{n}, 0\right), \quad \xi=\bar{\chi}\left(x^{1}, \ldots, x^{n}, 0\right) \eta=\left.\frac{\partial \bar{\chi}}{\partial y}\right|_{y=0}, \quad \phi\left(x^{1}, . ., x^{n}\right)=\bar{\phi}\left(x^{1}, \ldots, x^{n}, 0\right)
$$

satisfy the equations (14), (15) and (16).

$(\Leftarrow)$ Suppose that there exist functions $\Omega_{i k}\left(x^{1}, \ldots, x^{n}\right), \xi\left(x^{1}, . ., x^{n}\right), \eta\left(x^{1}, . ., x^{n}\right)$ and $\phi\left(x^{1}, . ., x^{n}\right) \neq 0$ which satisfy (14), (15) and (16). Choose an analytic function $\bar{\phi}\left(x^{1}, \ldots, x^{n}, y\right) \neq 0$ such that $\bar{\phi}\left(x^{1}, \ldots, x^{n}, 0\right)=$ $\phi\left(x^{1}, . ., x^{n}\right)$. By virtue of the CK theorem, there exists a unique set of analytic functions $\bar{g}_{i k}\left(x^{1}, \ldots, x^{n}, y\right)$ and $\bar{\chi}\left(x^{1}, \ldots, x^{n}, y\right)$ that satisfy the equations (4) and (6) and the initial conditions (13). Since, by assumption, the initial conditions satisfy equations (14), (15) and (16), equations (6) and (7) are satisfied at $y=0$ by $\bar{g}_{i k}, \bar{\phi}$ and $\bar{\chi}$. It follows from Lemma 1 that $\bar{g}_{i k}, \bar{\phi}$ and $\bar{\chi}$ also satisfy equations (4), (6) and (17) in an open set of $\Re^{n+1}$ which contains the origin. We conclude, therefore, that the $(n+1)$-dimensional manifold whose line element (3) is expressed in terms of the solutions $\bar{g}_{i k}$ and $\bar{\phi}$ is a space generated by a scalar field. Thus, the (semi-) Riemannian manifold $\left(M^{n}, g\right)$ can indeed be embedded in a space sourced by a scalar field.

According to Theorem 1, the existence of solutions to equations (14), (15) and (16) is sufficient to ensure that the local, analytic embedding of $M^{n}$ is possible. The proof that these solutions do in fact exist consists in showing that equations (14), (15) and (16) can be expressed in the canonical form required by the CK theorem (here in its first-derivative version). This can be done by following the method presented in [8] with no significant modifications, and so we omit the details here. The idea is to use (16) to isolate $\Omega_{11}$ which is then substituted into (15) which is to be regarded as a system of p.d.e's for $n$ unknown functions: the $n-1 \Omega_{1 k}$ for $k \geq 2$ and one other component of $\Omega$, which we call $\Theta$. These p.d.e's are in the correct canonical form to employ the CK theorem. Thus, if the analytic functions $g_{i k}$ are given, there exist analytic functions $\Omega_{i k}$ which solve equations (14), (15) and (16) and this leads us to the following theorem:

Theorem 2. Let $M^{n}(n>1)$ be a piece of a (semi-)Riemannian space with line element $d s^{2}=g_{i k} d x^{i} d x^{k}$, expressed in a coordinate system which covers a neighborhood of a point $p \in M^{n}$ whose coordinates are $x_{p}^{1}=\ldots=$ $x_{p}^{n}=0$. If $g_{i k}$ are analytic functions at $0 \in \Re^{n}$, then $M^{n}$ can be embedded at $p$ in some $(n+1)$-dimensional space sourced by any arbitrary scalar field satisfying the conditions (2), (4) and (5). Moreover, the line element of the embedding space is unique if the arbitrary functions to be chosen obey the following conditions:

i) the $[n(n-1) / 2]-1$ functions $\Omega_{i k}(i \leq k, i>1$, and excluding the component $\Theta)$ are analytic at $0 \in \Re^{n}$;

ii) the $n$ functions $\Omega_{1 k}\left(0, x^{2}, \ldots, x^{n}\right)=f_{k}\left(x^{2}, \ldots, x^{n}\right)(k>1)$ and $\Theta\left(0, x^{2}, \ldots, x^{n}\right)=f_{1}\left(x^{2}, \ldots, x^{n}\right)$ are analytic at $0 \in \Re^{n-1}$, with the coefficient of $\Omega_{11}$ in (16) nonzero (to permit the elimination of $\Omega_{11}$ in order to set up the p.d.e system),

iii) a function $\phi\left(x^{1}, \ldots, x^{n+1}\right) \neq 0$, analytic at $0 \in \Re^{n+1}$, is chosen;

iv) two functions $\xi\left(x^{1}, \ldots, x^{n}\right)$ and $\eta\left(x^{1}, \ldots, x^{n}\right)$, analytic at $0 \in \Re^{n}$, are chosen.

Note that when we refer to a space generated by a scalar field we have in mind a non-trivial solution of the Einstein-scalar system. In other words, we are implicitly considering a solution such that the associated energy-momentum tensor is nonzero. The exclusion of trivial solutions is possible because the initial conditions of the field (the functions $\xi$ and $\eta$ ) are arbitrary and this implies that they can be chosen in such a way that $T_{\alpha \beta} \neq 0$ at $\Sigma_{0}$ (for some $\alpha$ and $\beta$ ). Consequently, continuity requirements imply that the energy-momentum tensor does not vanish in some neighborhood of $0 \in \Re^{n+1}$.

We proceed in the following Section to employ this theorem to establish specific classes of embeddings.

\section{Applications}

\subsection{Self-interacting scalar fields}

In this section we consider a real scalar field, $\chi$, minimally coupled to Einstein gravity and self-interacting through a potential, $W(\chi)$. We assume that the potential is a well-behaved, analytic function of the field. The 
energy-momentum tensor of such a field is given by

$$
T_{\alpha \beta}=\nabla_{\alpha} \chi \nabla_{\beta} \chi-\frac{1}{2} g_{\alpha \beta}\left(\nabla^{\gamma} \chi \nabla_{\gamma} \chi\right)-g_{\alpha \beta} W(\chi) .
$$

It easily follows that the energy-momentum tensor (18) has vanishing divergence if $\chi$ solves the field equation

$$
\nabla^{\alpha} \nabla_{\alpha} \chi-\frac{d W}{d \chi}=0
$$

In the coordinate system (3) the field equation (19) takes the form of equation (41) with

$$
P=\varepsilon \phi^{2}\left(-g^{i k} \frac{\partial^{2} \chi}{\partial x^{i} \partial x^{k}}-g^{\alpha \beta} \Gamma_{\alpha \beta}^{\gamma} \frac{\partial \chi}{\partial x^{\gamma}}+\frac{d W}{d \chi}\right) .
$$

Note that the function $\mathrm{P}$ which depends on $\chi, \frac{\partial \chi}{\partial x^{i}}, \frac{\partial \chi}{\partial y}, g_{\alpha \beta}, \frac{\partial g_{\alpha \beta}}{\partial x^{i}}, \frac{\partial g_{\alpha \beta}}{\partial y}$, and $\frac{\partial^{2} \chi}{\partial x^{i} \partial x^{k}}$, is analytic with respect to each of these arguments.

Since conditions (21), (4) and (5) are satisfied by a minimally coupled, self-interacting scalar field, we may conclude the following:

Corollary 1. Let $M^{n}(n>1)$ be a piece of a (semi-)Riemannian space with line element $d s^{2}=g_{i k} d x^{i} d x^{k}$, expressed in a coordinate system which covers a neighborhood of a point $p \in M^{n}$ whose coordinates are $x_{p}^{1}=$ $\ldots=x_{p}^{n}=0$. If $g_{i k}$ are analytic functions at $0 \in \Re^{n}$, then $M^{n}$ can be embedded at $p$ in some $(n+1)$-dimensional space generated by any arbitrary, minimally coupled, self-interacting scalar field.

Note that $M^{n}$ is truly Riemannian for $\varepsilon=-1$ and semi-Riemannian for $\varepsilon=+1$.

\subsection{Examples of Embeddings with Self-Interacting Scalars}

We now employ this result to construct embeddings for a class of space-times into higher-dimensional space-times sourced by such a scalar field. We begin with the ansatz

$$
\bar{\Omega}_{i j}=C \bar{g}_{i j}
$$

where $C=C\left(x^{\alpha}\right)$ is a scalar function of the coordinates of the embedding metric. We further assume that the scalar field is independent of the embedded metric coordinates, i.e., $\chi=\chi(y)$, and specify $\kappa=1$ and $\varepsilon=1$ for simplicity.

Substituting (21) into (15) implies that $\nabla_{i} C=0$ and, consequently, that $C$ must be a function of the extra coordinate $y$ alone. Choosing the normal coordinate form for $\phi$ :

$$
\phi=1,
$$

then implies that (8) can be integrated to yield the solution

$$
\bar{g}_{i j}=a^{2}(y) g_{i j}
$$

where $C \equiv-d \ln a / d y$.

When (21) and (22) are valid, the scalar field equation (19) simplifies to

$$
\frac{d^{2} \chi}{d y^{2}}+\frac{n}{a} \frac{d a}{d y} \frac{d \chi}{d y}-\frac{d V}{d \chi}=0
$$

and by substituting the appropriate components of the energy-momentum tensor (18) into (6) and (7), we find that

$$
\begin{array}{r}
\bar{R}_{i k}+\bar{\Omega} \bar{\Omega}_{i k}-2 \bar{\Omega}_{i m} \bar{\Omega}_{k}^{m}-\frac{\partial \bar{\Omega}_{i k}}{\partial y}=-\frac{2 V}{n-1} \bar{g}_{i k} \\
\bar{R}+\bar{\Omega}^{2}-\bar{\Omega}_{j}^{i} \bar{\Omega}_{i}^{j}=\left(\frac{d \chi}{d y}\right)^{2}-2 V .
\end{array}
$$

Furthermore, subtracting (26) from the trace of (25) and substituting for the ansatz (21) implies that

$$
\frac{n}{a} \frac{d^{2} a}{d \nu^{2}}=-\left(\frac{d \chi}{d u^{\prime}}\right)^{2}-\frac{2 V}{n-1} .
$$


We may solve (27) and (24) for an unknown self-interaction potential, by specifying the functional forms of $a(y)$ and $\chi(y)$ :

$$
\begin{gathered}
a \equiv(1+\lambda y)^{p}, \\
\chi \equiv q \ln (1+\lambda y),
\end{gathered}
$$

where $p, q$ and $\lambda$ are constants. Substituting (28) and (29) into (27) implies that this is consistent if the scalar field potential has an exponential (Liouville) form:

$$
V=-\lambda^{2}\left(\frac{n-1}{2}\right)\left[n p(p-1)+q^{2}\right] \exp \left(-\frac{2}{q} \chi\right)
$$

and it then follows that the scalar field equation (24) is solved if

$$
(p-1)\left[q^{2}-p(n-1)\right]=0 .
$$

Thus, the embedding is established by solving (25) for the two cases, where $q^{2}=p(n-1)$ or $p=1$. In the first case, substituting for (28), (29) and (30) implies that the embedding metric, $g_{i j}$, must have vanishing Ricci tensor. We conclude, therefore, that there is an embedding of $n-\mathrm{d}$ Ricci-flat manifolds with metric $g_{i j}$, in an $(n+1)-\mathrm{d}$ manifold sourced by a scalar field, with metric given by

$$
d s^{2}=(1+\lambda y)^{p} g_{i k} d x^{i} d x^{k}+d y^{2},
$$

where the scalar field (29) self-interacts through the exponential potential (30). This generalizes the 4-d result of [29] to arbitrary dimension.

In the second case, where $p=1$, the above procedure implies that (25) is solved if

$$
R_{i k}=\frac{\left(q^{2}-n+1\right) \lambda^{2}}{(1+\lambda y)^{2}} g_{i k}
$$

and it follows that the embedded metric is an Einstein space with a non-zero Ricci curvature. The sign and magnitude of the effective cosmological constant of the embedded manifold determine the self-interaction coupling, $q$, of the scalar field. Indeed, the potential (30) is negative-definite for this embedding. An embedding of this type has been considered within the context of dilatonic braneworld scenarios [30. It is interesting that the functional form of the potential (30) is the same for the two different classes of embedding. Moreover, potentials of this form arise in compactified supergravity theories [31].

The above embeddings are specific in the sense that for the assumed form of $\Omega_{i k}$ given in (21), the embedding is only consistent if the scalar field has an exponential potential. However, Corollary 1 states that an embedding is possible for any analytic potential. This would require some other form for $\Omega_{i k}$ to be chosen.

\subsection{Brans-Dicke Theory}

The Brans-Dicke theory of gravity [20] represents a natural extension of GR, where a nonminimally-coupled ('dilatonic') scalar field parametrizes the space-time dependence of Newton's 'constant'. Nevertheless, it is well-known that this theory is conformally equivalent to GR with a minimally-coupled scalar field. Therefore, the following corollary to the above theorems is deduced if one is prepared to work within the context of the conformally-transformed fields.

Corollary 2. Let $M^{n}(n>1)$ be a piece of a (semi-)Riemannian space with line element $d s^{2}=g_{i k} d x^{i} d x^{k}$, expressed in a coordinate system which covers a neighborhood of a point $p \in M^{n}$ whose coordinates are $x_{p}^{1}=\ldots=$ $x_{p}^{n}=0$. If $g_{i k}$ are analytic functions at $0 \in \Re^{n}$, then $M^{n}$ can be embedded at $p$ in some $(n+1)$-dimensional space which is a solution of the vacuum, Brans-Dicke field equations.

\section{Campbell-Magaard and the GR Cauchy and Initial Value Problems}

In Sec 2, we referred to the close relationship between the CM theorem and the GR Cauchy and initial value problems (CP and IVP, respectively). There is a wealth of literature on the latter problems within the context of $(3,0) \longrightarrow(3,1)$ embeddings $^{2}$, which makes it a valuable source of ideas for embedding theorems. The first

\footnotetext{
${ }^{2}$ We denote by $(p, q)$ a manifold with $p$ spacelike and $q$ timelike coordinates.
} 
idea is to show how the CM theorem follows from a collection of known results. The second idea will be to question whether any of the dimension- or signature-dependent results of the GR CP have useful generalizations. This will lead to possible limitations of the usual methods of constructing higher-dimensional solutions from lower-dimensional ones. We begin by outlining the GR CP and IVP.

By use of the $(3+1)$ split of the space-time line element ${ }^{3}$

$$
d s^{2}=h_{i j}\left(N d x^{i}+N^{i} d t\right)\left(N d x^{j}+N^{j} d t\right)-(N d t)^{2}
$$

the 10 EFE's may be rewritten as 6 evolution equations and the 4 Gauss-Codazzi constraints. The mathematics of this split is the same, up to the signature, as that of splitting the EFE's into equation (6) and into equations (7) and (8), if one identifies the lapse to be $\phi$ and imposes the partial gauge condition that the shift be zero. The GR CP is then the study of the evolution equations given some initial data that obeys the constraints.

It is standard knowledge [21, 22] that these evolution equations can be written in the correct form required by the CK theorem, and so we are guaranteed that a solution exists locally and that it is unique. Whereas self-consistency requires the evolution equations to propagate the constraints off the initial hypersurface, it is immediately evident that this follows from the Bianchi identities.

There are additional results about the GR CP having a number of physically-desirable features. Firstly, it is Hadamard well-posed [27, 21] so that in addition to the existence of a unique solution, the solution depends continuously on the prescribed data. Without this, an arbitrarily small change in the data set could give rise to an arbitrarily large change in the form of the solution which prevents physical predictability. Secondly, it possesses the 'domain of dependence' property, i.e., the data can only affect the evolution in regions that are in causal contact with that data, which is a necessary criterion for the good behaviour of hyperbolic systems 21, 23. The continuous dependence and domain of dependence properties follow in harmonic coordinates from Leray's theorem [32, 21], which is specific to hyperbolic operators. Finally, it is standard knowledge that the GR CP is well-posed in the presence of scalar fields, electromagnetism, perfect fluids, Brans-Dicke theory and certain higher-derivative theories [21].

The IVP or data construction problem (solving the Gauss-Codazzi constraints) is considered to be the most difficult step in the $(3+1)$ formalism of GR [33]. This problem is under-determined, because there are 12 unknown functions (the components of the 3 -metric $h_{i k}$ and the second fundamental form $\Omega_{i k}$ ), but only 4 equations. Hawking and Ellis 34] apparently refer to a general result stating that if eight of the unknown functions are specified for a space-time with an arbitrary matter content, the constraint equations may be solved for the remaining four. However, the work they refer to, Ref. [22], considers only two methods (the thin sandwich method and the usual conformal method), neither of which resemble Magaard's method.

We now consider the CM theorem piece by piece. The structure of none of the above results are dependent on the dimension. Neither the CK theorem nor the method of expressing the GR evolution equations in the correct form to invoke the CK theorem are affected by the signature. Furthermore the Bianchi identity which guarantees constraint propagation is geometrical and hence equally valid regardless of the signature. Hence, the first part of the proof of the CM theorem (existence, uniqueness and constraint propagation) follows directly from these results holding for the $(3+1)-\mathrm{d}$ GR CP.

The second part of the proof, due to Magaard, is a subcase of the result in Hawking and Ellis [34 generalized to arbitrary dimension. However, we emphasize that Magaard's approach differs from that of the usual conformal data construction [24, 25, 26]. The former considers the lower-dimensional metric to be a known function, but the latter considers it to be known only up to a conformal factor. The former is of interest following the new motivation for studying higher-dimensional space-times, whereas the latter has only been employed for three spacelike dimensions using largely signature-dependent (that is, elliptical) methods.

If we are to interpret the CM theorem from a physical point of view, we are well-motivated to demand that the procedure for constructing embeddings is a well-posed problem. The types of embeddings of interest are $(n, 1) \longrightarrow(n+1,1)$ and $(n, 1) \longrightarrow(n, 2)$, which are forms which are not known to exhibit continuous dependence, respectively ${ }^{4}$. The former is termed the 'sideways problem for a hyperbolic system', and arises in a number of non-relativistic contexts, as summarized by Ames and Straughan [35. The only general result known for simple examples of such problems is that, given certain bounds, the solution is Holder-stable in some region [36], but this is not considered to amount to a sufficiently strong theorem to guarantee continuous dependence. The latter

\footnotetext{
${ }^{3}$ Here, $N$ is the lapse, $N^{i}$ is the shift and $h_{i j}$ is the induced 3-metric.

${ }^{4}$ There is no simple way of investigating whether theorems that hold for one particular signature hold for any other. This is due to significant mathematical differences between elliptic and 'hyperbolic' operators. ¿From the point of view of the function spaces that underly the analysis, Holder spaces are natural for the study of elliptic operators while Sobolev spaces are natural for the study of hyperbolic operators.
} 
is an ultrahyperbolic problem. These remain largely unexplored since they were traditionally considered to be physically irrelevant (see however [37]). This implies, for example, that there are problems with the physical interpretation of models based on GR with two timelike dimensions [38. (as also pointed out in $(10+2)-\mathrm{d}$ supergravity [39]).

If we exclude this second possibility, it may be more promising to approach higher-dimensional embeddings with a two-step procedure of the form $(3,0) \longrightarrow(4,0) \longrightarrow(4,1)$. The first step could be a boundary problem along the lines considered in [40, while the second step is a higher-dimensional version of the GR CP. This procedure would allow the bulk to influence the hypersurface in a causal way (in the usual GR sense), which is not possible in a $(3,1) \longrightarrow(4,1)$ type of embedding (except perhaps in a perturbative treatment). It is clearly of interest to construct models in which the bulk does play a role that is, in principle, observable and testable. Although the nature of causality in higher-dimensional theories may be substantially different to that of $(3+1)-\mathrm{d}$ GR 41, such models would be consistent with a direct extension of GR.

\section{Conclusion}

In this paper, we have proved an extension of the CM embedding theorem [18, 19, where the embedding metric is sourced by one or more scalar fields minimally coupled to GR. We employed this theorem to establish classes of embeddings where the scalar field self-interacts through an exponential potential. The relationship between the CM theorem and the Cauchy and initial value problems of GR was highlighted. This relationship will certainly permit CP an IVP techniques to be adapted to provide further space-time into space-time embeddings.

Embedding theorems are important from both the mathematical and physical points of view. They allow classification schemes for different spacetimes to be developed as well as providing algorithms for generating new solutions [42. The primary physical motivation for developing mathematical embedding theorems arises from the resurgence of interest in higher-dimensional theories of gravity and cosmology, most notably the braneworld scenarios [3, 4, 5, 6]. In particular, the Randall-Sundrum 'type II' (RSII) scenario [6], where a codimension one brane is embedded in five-dimensional Anti-de Sitter $\left(\mathrm{AdS}_{5}\right)$ space, has attracted considerable attention. Embeddings are also of relevance to the induced 'space-time-matter' (STM) theory, whereby the 4-d universe is viewed as a slice of a 5-d universe [43, 44, 17]. (For reviews, see, e.g., Refs. [12, 45]). The CM theory was recently employed within the context of both the RSII scenario and STM theory where the close similarities between the two approachs were also highlighted and algorithms for employing the theorem in practice were outlined [17. The relationship between the two pictures has also been emphasized in Ref. [44.

Although the RSII model was motivated in part by the Horăva-Witten theory for the strongly coupled limit of the $\mathrm{E}_{8} \times \mathrm{E}_{8}$ heterotic string theory [4, it is an idealized system, in the sense that the higher-dimensional space is assumed to be an Einstein space. It is therefore important to consider more general settings that are inspired by string theoretic considerations. For example, the supergravity actions contain, in addition to the scalar dilaton field, a number of antisymmetric form-fields. Up on compactification from ten or eleven dimensions, the degrees of freedom associated with these fields can manifest themselves as scalar fields that self-interact through one or more potentials. In particular, scalar fields arise when the form fields have a non-trivial flux over the compactifying dimensions that is able to support a solitonic brane configuration. A specific example is the compactification of the Horăva-Witten theory to five dimensions [ $\underline{5}$, where a scalar field arises in the fifth (bulk) dimension through an exponential potential of the form considered in Sec 3.2.

In view of the above discussion, it is important to develop the mathematical framework for studying braneworld models containing scalar fields in the higher dimensions through embedding theorems. Unlike almost all ${ }^{5}$ current analyses, our treatment in the present work is for the full Einstein-scalar field system rather than for some highly symmetric case. This general treatment is appropriate because we are establishing an existence and uniqueness theorem for embeddings into space-times sourced by scalar fields.

It is worth emphasizing that the scalar fields we have considered in our extension of the CM theorem are additional, dynamical bulk scalar fields, $\bar{\chi}$, and not the higher-dimensional metric component, $\bar{\phi}$. The theorem we have proved therefore provides a starting point for considering a number of open physical questions, such as the possible restrictions that may arise on the energy-momentum tensor of the brane matter fields once the junction conditions are imposed. A related question is whether plausible $4-\mathrm{d}$ physics can generically be recovered and whether there are testable corrections to Newton gravity such as those in the RSII scenario [6]. It would be interesting to consider these questions further.

Finally, our generalization in this paper of the CM theorem implies that for a given 4-d space-time, there are an infinite number of embedding space-times with one extra dimension, in the sense that there is at least one

${ }^{5}$ This is also the starting point of the perturbative $4-\mathrm{d}$ treatment of domain walls presented in [46]. 
possible embedding for each functional form of the higher-dimensional energy-momentum tensor ${ }^{6}$, in particular, one per scalar interaction potential of the surrounding 5 -d space-time. This is arguably an undersirable feature for higher-dimensional theories, since it implies that unique physical predictions can not be made in the absence of well-founded principles that select a particular higher-dimensional space-time. This is similar to the wellknown problem in string theory phenomenology, where different particle spectra arise for different Calabi-Yau compactifications [47. The introduction of discontinuities or branes is unlikely to remove this non-uniqueness property, since there are many possible brane configurations (single, parallel or intersecting) that could be considered and many possible forms for the higher-dimensional bulk space-time, but presently no established way to distinguish between them from a physical point of view. Perhaps, the number of fields and the form of the potential would be restricted in some higher-dimensional theory and by requiring the recovery of (possibly corrected) 4-d physics as discussed above. Our theorem provides a framework within which these and related questions can be addressed.

\section{Acknowledgements}

EA is supported by PPARC. FD and CR are supported by CNPq. JEL is supported by the Royal Society. We thank M. A. H MacCallum, N. Ó. Murchadha and R. Tavakol for helpful discussions and communications.

\section{References}

[1] E. Witten, Nucl. Phys. B 443, 85 (1995); P. Townsend, Phys. Lett. B 350, 184 (1995).

[2] J. Polchinski, String Theory (Cambridge University Press, Cambridge, 1998); C. V. Johnson, D-Branes (Cambridge University Press, Cambridge, 2003).

[3] K. Akama, hep-th/0001113 V. A. Rubakov and M. E. Shaposhnikov, Phys. Lett. B 159, 22 (1985); M. Visser, Phys. Lett. B 159, 22 (1985); N. Arkani-Hamed, S. Dimopoulos, and G. Dvali, Phys. Lett. B 429, 263 (1998); I. Antoniadis, N. Arkani-Hamed, S. Dimopoulos, and G. Dvali, Phys. Lett. B436, 257 (1998); L. Randall and R. Sundrum, Phys. Rev. Lett. 83, 3370 (1999); M. Gogberashvili, Europhys. Lett. 49, $396(2000)$.

[4] P. Hořava and E. Witten, Nucl. Phys. B 460, 506 (1996); P. Hořava and E. Witten, Nucl. Phys. B 475, 94 (1996).

[5] A. Lukas, B. A. Ovrut, K. S. Stelle, and D. Waldram, Phys. Rev. D 59, 086001 (1999); A. Lukas, B. A. Ovrut, and D. Waldram, Phys. Rev. D 60, 086001 (1999).

[6] L. Randall and R. Sundrum, Phys. Rev. Lett. 83, 4690 (1999).

[7] M. Pavsic and V. Tapia, gr-qc/0010045

[8] C. Romero, R. Tavakol and R. Zalaletdinov, Gen. Rel.Grav. 28, 365 (1996)

[9] M. D. Maia, gr-qc/9512002

[10] J. E. Lidsey, C. Romero, R. Tavakol, S. Rippl, Class. Quantum Grav. 14, 865 (1997).

[11] J. E. Lidsey, Phys. Lett. B 417, 33 (1997).

[12] P. S. Wesson, Space-Time-Matter (World Scientific, Singapore, 1999).

[13] A. G. Agnese and M. La Camera, Nuovo Cim. B115, 119 (2000).

[14] E. Anderson and J. E. Lidsey, Class. Quantum Grav. 18, 4831 (2001).

[15] F. Dahia and C. Romero, J. Math. Phys. 43, 3097 (2002).

[16] F. Dahia and C. Romero, J. Math. Phys. 43, 5804 (2002).

[17] S. S. Searha and P. S. Wesson, Class. Quantum Grav. 20, 1321 (2003).

[18] J. E. Campbell, A Course of Differential Geometry (Claredon, Oxford, 1926).

[19] L. Magaard, Zur einbettung riemannscher Raume in Einstein-Raume und konformeuclidische Raume (PhD Thesis, Kiel, 1963).

[20] C. Brans and R. Dicke, Phys. Rev. 124, 925 (1961); R. Dicke, Phys. Rev. 125, 2163. (1962).

[21] R. M. Wald, General Relativity (Chicago University Press, Chicago 1984).

[22] Y. Bruhat, Gravitation: An introduction to current research ed. L. Witten (Wiley, New York, 1962).

[23] S. W. Hawking and G. F. R. Ellis The Large-scale Structure of Spacetime (Cambridge University Press, Cambridge, 1973).

\footnotetext{
${ }^{6}$ In the embedding procedure, the energy-momentum tensor is viewed as a function of the coordinates and not as a function of the fields. This implies that the higher-dimensional models are merely encoding solutions rather than specific physical laws. Compare with how Kaluza-Klein theory offered further predictions as a result of the geometrization of the electromagnetic field.
} 
[24] A. Lichnerowicz, J. Math. Pures Appl. 2337 (1944).

[25] J. W. York, Phys. Rev. Lett. 26, 1657 (1971); J. W. York, Phys. Rev. Lett. 28, 1082 (1972); J. W. York, J. Math. Phys. 14, 456 (1973).

[26] J. W. York and Y. Choquet-Bruhat, General Relativity and Gravitation Vol 1 ed. A. Held (Plenum Press, New York, 1980).

[27] See, for example, R. Courant and D. Hilbert, Methods of Mathematical Physics Vol. II (Wiley, New York, 1989).

[28] F. Dahia, Imersão do espaco-tempo e a generalizacão do teorema de Campbell-Magaard (PhD thesis, UFPB, Brazil, 2001).

[29] J. E. Lidsey, Class. Quantum Grav. 17, 453 (2000); A. Feinstein, K. E. Kunze, and M. A. Vazquez-Mozo, Phys. Rev. D 64, 084015 (2001).

[30] N. Alonso-Alberca, B. Janssen, and P. J. Silva, Class. Quantum Grav. 17, L163 (2000).

[31] H. Lü, C. N. Pope, and P. K. Townsend, Phys. Lett. B 391, 39 (1997); P. M. Cowdell, H. Lü, C. N. Pope, K. S. Stelle, and P. K. Townsend, Nucl. Phys. B 486, 49 (1997).

[32] J. Leray, Hyperbolic Partial Differential Equations (Institute for Advanced Study, 1955).

[33] C. W. Misner, K. Thorne, and J. A. Wheeler, Gravitation (Freedman, San Francisco, 1973) page 539.

[34] S. W. Hawking and G. F. R. Ellis, The Large-scale Structure of Spacetime (Cambridge University Press, Cambridge, 1973 ), page 232.

[35] K. A. Ames and B. Straughan, Non-Standard and Improperly Posed Problems (Academic press, San Diego, 1997).

[36] K. A. Ames and V. J. Isakov, Math. Anal. Appl. 156, 597 (1991).

[37] M. M. Lavrent'ev, V. G. Romanov and, S. P. Shishatskii, Ill-Posed Problems of Mathematical Physics and Analysis, Translations of Mathematical Monographs 64, (American Mathematical Society, Providence, Rhode Island, 1986).

[38] M. Chaichian and A. B. Kobakhidze, Phys. Lett. B 488, 117 (2000); Z. Berezhiani, M. Chaichian, A. B. Kobakhidze, and Z. H. Yu, Phys. Lett. B 517, 387 (2001).

[39] S. F. Hewson, hep-th/9908209

[40] O. Reula, Class. Quantum Grav. 5, 179 (1988).

[41] D. J. H. Chung and K. Freese, Phys. Rev. D 62, 063513 (2000); R. R. Caldwell and D. Langlois, Phys. Lett. B 511, 129 (2001); C. Csaki, J. Erlich, and C. Grojean, Nucl. Phys. B 604, 312 (2001).

[42] D. Kramer, H. Stephani, E. Herlt, and M. A. H. MacCallum, Exact Solutions of Einstein's Field Equations (Cambridge, Cambridge University Press, 1980).

[43] P. S. Wesson, Gen. Rel. Grav. 16, 193 (1984); J. Ponce de Leon, Gen. Rel. Grav. 20, 539 (1988); P. S. Wesson and J. Ponce de Leon, J. Math. Phys. 33, 3883 (1992); P.S. Wesson, Phys. Lett. B 276, 299 (1992); P. S. Wesson et al., Int. J. Mod. Phys. A11, 3247 (1996); A. A. Coley and D. J. McManus, J. Math. Phys. 36, 335 (1995); A. P. Billyard and A. A. Coley, Mod. Phys. Lett. A12, 2121 (1997); W. N. Sajko and P. S. Wesson, Mod. Phys. Lett. A16, 627 (2001); A. P. Billyard and W. N. Sajko, Gen. Rel. Grav. 33, 1929 (2001); J. Ponce de Leon, Mod. Phys. Lett. A16, 1405 (2001); M. Bellini, Nucl. Phys. B660, 389 (2003).

[44] J. Ponce de Leon, Mod. Phys. Lett. A16, 2291 (2001).

[45] J. M. Overduin and P. S. Wesson, Phys. Rept. 283, 303 (1997).

[46] F. Bonjour, C. Charmousis, and R. Gregory, Phys. Rev. D62, 083504 (2000).

[47] See, for example, M. Kaku, Introduction to Superstrings (Springer-Verlag, New York, 1988). 